\title{
GNSS multi-satellite joint acquisition with interference mitigation using antenna arrays
}

\author{
Gangqiang Guan ${ }^{1, a}$, Yangbo Huang ${ }^{1}$, Baiyu $\mathrm{Li}^{1}$ and Feixue Wang ${ }^{1, b}$ \\ ${ }^{1}$ College of Electronic Science and Engineering, National University of Defense Technology, \\ Changsha, China \\ aclosetoqiang@163.com, bfeixuewang_nnc@163.com
}

Keywords: GNSS acquisition, interference mitigation, adaptive filter, antenna array, multi-satellite.

\begin{abstract}
In this letter the antenna array based GNSS signal acquisition is proposed by firstly projecting the input signal vector onto the noise subspace which is orthogonal to interference subspace for strong interferences suppression. Then, considering that the projection matrix is rank-deficient, we propose to apply forward spatial smoothing to decorrelation of the coherent interference-free signal vector and a whitening filter to prewhitening of the spatial colored noise. Then the signal vector is fed to an adaptive multi-satellite acquisition and beamforming processor. This processor simultaneous performs adaptive beamforming and multi-satellite code acquisition by a spatial filter and a local code select filter, while the order of select filter corresponds to the number of satellites searched in a dwell cell. When the desired satellite signal is present in the test cell, the peak weight of the select filter will converge to the local code of the right satellite signal while the weights of the spatial filter will converge to the direction of arrival (DOA) estimation respectively. If not, the spatial weights will converge to zeros and the local select filter weights will be an arbitrary vector which satisfies the additional constraint, then the next cell is tested and the adaptive process is repeated.
\end{abstract}

\section{Introduction}

In the open literature a number of papers on code acquisition in direct-sequence code-division multiple-access (DS-CDMA) systems can be found. In [1-2] similar approaches with minimum mean squared error (MMSE) criterion are proposed combing adaptive temporal filtering and beamforming for acquisition utilizing the desired user's local pseudo-noise (PN) code as the reference signal. In [3-4] code acquisition is all essentially accomplished using a full serial search strategy which is time-consuming. However, considering the extremely low power of GNSS signal and strong interferences situation the above scheme for beamformer and acquisition can't be guaranteed. Up to now fewer papers appeared on GNSS signal acquisition based on antenna array, in [5] a GNSS signal acquisition approach with interference mitigation based on Neyman-Pearson (NP) and generalized likelihood ratio test (GLRT) detection theory is proposed from statistical perspective and the performance is analyzed theoretically. In [6] a space-time adaptive processing (STAP) algorithm using extended Kalman filter (EKF) for code acquisition detection and interference rejection is presented. However these proposed methods for signal acquisition have a common problem of great computational complexity.

This letter proposes a novel GNSS multi-satellite joint acquisition scheme using antenna array. The key point of this scheme is that it can perform multi-satellite joint acquisition and adaptive beamforming in strong interference and low signal to noise ratio (SNR) environment while the acquired satellite is distinguishable, and furthermore the computation complexity and convergence rate are acceptable. 


\section{Signal Model}

Suppose the signal impinging on each element of the array consists of GNSS signal, interference and additive white Gaussian noise (AWGN), the complex baseband signal vector received by $\mathrm{N}$ antenna elements after AD convertor and digital down conversion (DDC) can be expressed as

$$
\mathbf{x}(n)=\mathbf{A} s(n)+\mathbf{B} i(n)+\boldsymbol{\eta}(n)
$$

where $\mathbf{A}=\left[\mathbf{a}_{1}, \ldots, \mathbf{a}_{M}\right]_{N \times M}, \mathbf{B}=\left[\mathbf{b}_{1}, \ldots, \mathbf{b}_{L}\right]_{N \times L}$ are the steering matrices of $\mathbf{M}$ satellites and $\mathrm{L}$ interference respectively. $\mathbf{s}(n)=\left[s_{1}(n), \ldots, s_{M}(n)\right]_{M \times 1}^{T}, \quad \mathbf{i}(n)=\left[i_{1}(n), \ldots, i_{L}(n)\right]_{L \times 1}^{T}$ are the waveforms of signal and interference respectively, $\boldsymbol{\eta}(n)$ is the vector of AWGN with zero mean and $\sigma^{2} \mathbf{I}_{N \times N}$ variance matrix.

\section{Proposed Acquisition Scheme}

As can be seen in Fig.1, after projecting the baseband signal vector onto the noise subspace using subspace tracking method for interference mitigation, the signal vector is spatial correlated because the projection matrix is rank-deficient. Then through a spatial smoothing and prewhitening filter, an adaptive select weight vector $\boldsymbol{\omega}_{C}$ to combine the local PN code of P satellites $\mathbf{c}(n-\tau)$ as the reference signal $r(n)$ and an adaptive spatial weight vector $\omega_{S}$ for beamforming are utilized according the minimum mean square error (MMSE) criterion.

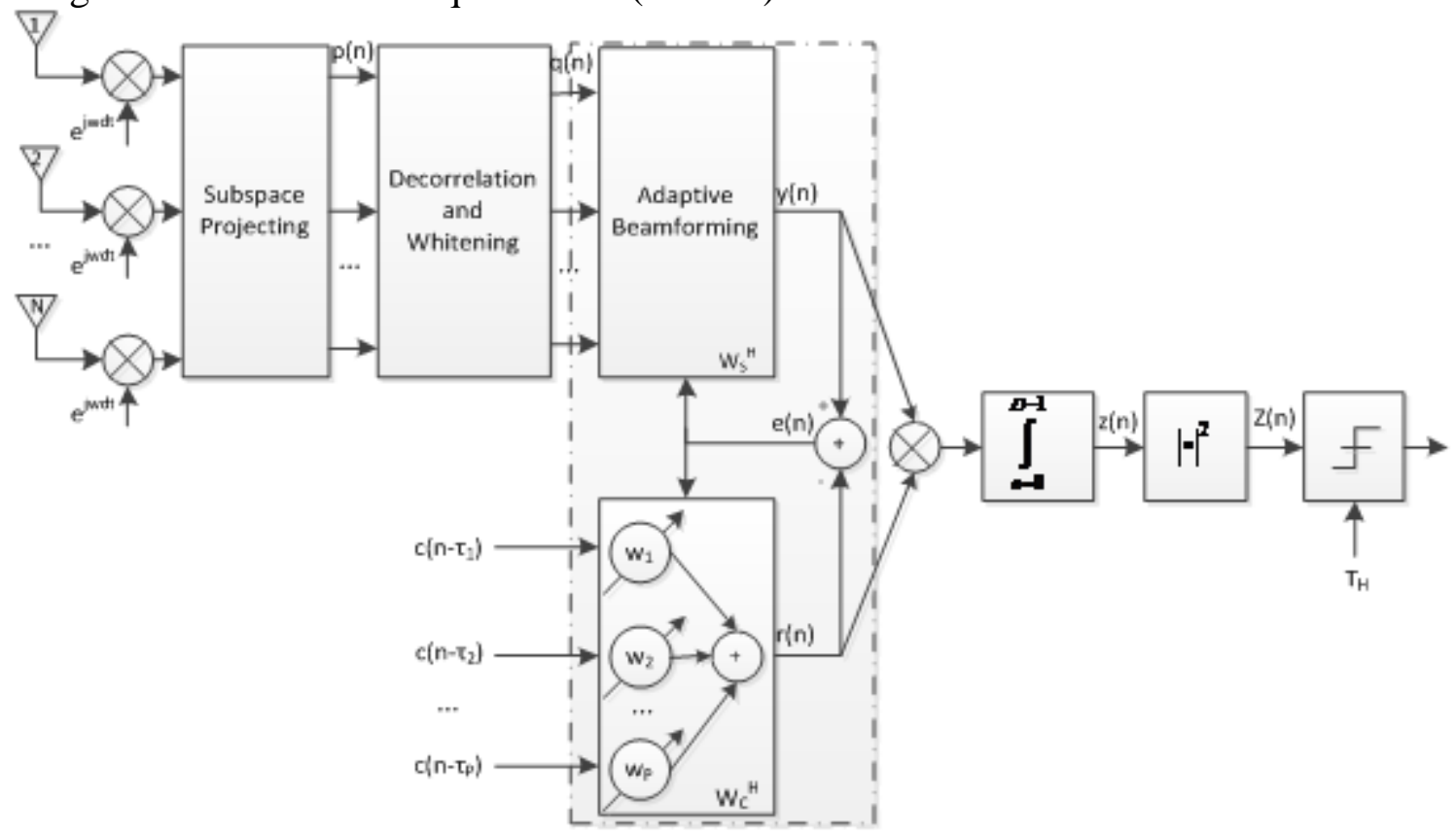

Fig. 1 Block diagram of the proposed GNSS acquisition scheme

That is, we choose $\boldsymbol{\omega}_{C}$ and $\omega_{S}$ to minimize the cost function

$$
J\left(\boldsymbol{\omega}_{C}, \boldsymbol{\omega}_{S}\right)=E\left[|e(n)|^{2}\right]=E\left[|r(n)-y(n)|^{2}\right]=E\left[\left|\boldsymbol{\omega}_{C}^{H} \mathbf{c}(n-\tau)-\boldsymbol{\omega}_{S}^{H} \mathbf{q}(n-\tau)\right|^{2}\right]
$$

where $\mathbf{q}(n)$ and $y(n)$ are the beamformer input and output respectively. To avoid the undesired trivial solution that $\boldsymbol{\omega}_{C}=\boldsymbol{\omega}_{S}=\mathbf{0}$, a constraint should be imposed on the adaptive process such as $\boldsymbol{\omega}_{C}$ is a real vector and $\boldsymbol{\omega}_{C}^{T} \boldsymbol{\omega}_{C}=1$. Thus the minimization of (2) turns out to be a constrained optimization problem where the Lagrange multiplier method can be applied. Through a simple mathematic derivation, the optimal solutions of the weights meet

$$
\boldsymbol{\omega}_{S, o p t}=-\mathbf{R}_{Q}^{-1} \mathbf{K}^{H} \boldsymbol{\omega}_{\mathrm{C}, \text { opt }}
$$




$$
\begin{gathered}
{\left[\mathbf{R}_{C}-\mathbf{K} \mathbf{R}_{Q}^{-1} \mathbf{K}^{H}\right] \boldsymbol{\omega}_{C, \text { opt }}-\xi \boldsymbol{\omega}_{C, \text { opt }}=\mathbf{0}} \\
J\left(\boldsymbol{\omega}_{C}, \boldsymbol{\omega}_{S}\right)_{\min }=\boldsymbol{\omega}_{C, \text { opt }}^{H}\left[\mathbf{R}_{C}-\mathbf{K} \mathbf{R}_{Q}^{-1} \mathbf{K}^{H}\right] \boldsymbol{\omega}_{C, \text { opt }}=\xi
\end{gathered}
$$

where $\xi$ denotes the Lagrange multiplier, and $\mathbf{R}_{C}=E\left[\mathbf{c}(n-\tau) \mathbf{c}(n-\tau)^{H}\right]$, $\mathbf{R}_{q}=E\left[\mathbf{q}(n) \mathbf{q}(n)^{H}\right], \mathbf{K}=E\left[\mathbf{c}(n-\tau) \mathbf{q}(n)^{H}\right]$. We can see that the optimization solution of MMSE cost function is equal to the minimum eigenvalue of $\mathbf{R}_{C}-\mathbf{K} \mathbf{R}_{Q}^{-1} \mathbf{K}^{H}$, while the optimum weight vector $\boldsymbol{\omega}_{C, \text { opt }}$ is the corresponding eigenvector. Once $\boldsymbol{\omega}_{C, \text { opt }}$ is available, $\boldsymbol{\omega}_{\mathrm{S} \text {,opt }}$ can be found using .

Considering the situation that the local replica code delay and carrier Doppler are in phase with a GNSS signal in sight while out of phase with other satellite signals, as well as the cross-correlation between different PN codes can be negligible, the received signal vector after subspace projection, spatial smoothing and prewhitening can be written as

$$
\mathbf{q}(n)=\mathbf{G}_{W} \mathbf{G}_{D} \mathbf{P}_{J} \mathbf{x}(n)=\mathbf{G}_{M}\left[\mathbf{a s}_{k}(n)+\boldsymbol{\eta}(n)\right]
$$

where $\mathbf{a}, s_{k}(n)$ are the steering vector and waveform of the inphase signal respectively. $\mathbf{G}_{W}, \mathbf{G}_{D}$, $\mathbf{P}_{J}$ and $\mathbf{G}_{M}$ is the prewhitening matrix, spatial smoothing matrix, projection matrix and their multiplication respectively. Then the cross-correlation matrix $\mathbf{K}$ can be rewritten as

$$
\mathbf{K}=[\underbrace{0, \ldots 0}_{h-1},-\mathbf{G}_{M} \mathbf{a}, 0, \ldots 0]^{H}
$$

where nonzero part lies in the $h^{\text {th }}$ row of $\mathbf{K}$ where the local replica PN code $c_{h}(n-\tau)$ is synchronized with the signal $s_{k}(n)$. Considering $\mathbf{R}_{C}=\mathbf{R}_{Q}=\mathbf{I}$, then we can have

$$
\mathbf{R}_{C}-\mathbf{K R}_{Q}^{-1} \mathbf{K}^{H}=\operatorname{diag}\left(1, \ldots 1,1-\mathbf{a}^{H} \mathbf{G}_{M}^{H} \mathbf{G}_{M} \mathbf{a}, 1, \ldots 1\right)
$$

where $\operatorname{diag}(\bullet)$ is diagonal operator. From we can find that has the minimum eigenvalue is $1-\mathbf{a}^{H} \mathbf{G}_{M}^{H} \mathbf{G}_{M} \mathbf{a}$. Then the corresponding eigenvector can be derived as

$$
\boldsymbol{\omega}_{C, o p t}=[\underbrace{0, \ldots 0}_{h-1}, 1,0, \ldots 0]^{T}
$$

Substituting into we can have the optimum spatial weight as $\boldsymbol{\omega}_{\mathrm{s}, \text { opt }}=\mathbf{G}_{M} \mathbf{a}$. After the MMSE solution is available, the correlation value between the output of beamformer and the local code filter is calculated and dumped periodically. The correlator output can be written as

$$
z=\sum_{n=0}^{D-1} \boldsymbol{\omega}_{\mathrm{S}, o p t}^{H} \mathbf{q}(n) \mathbf{c}(n-\tau)^{H} \boldsymbol{\omega}_{\mathrm{C}, \text { opt }}=D \mathbf{a}^{H} \mathbf{G}_{M}^{H} \mathbf{G}_{M} \mathbf{a}+\mathbf{a}^{H} \mathbf{G}_{M}^{H} \mathbf{G}_{M} \mathbf{a} \sum_{n=0}^{D-1} \eta(n) c_{h}(n-\tau)
$$

where $D$ is called as the integration time or spread spectrum gain and usually $D \square 1$. Until this time after the coherent integral the power of desired signal firstly exceeds that of the background noise, why the acquisition can be achieved. When the local code phase is out of phase the cross-correlation matrix $\mathbf{K}$ is a null matrix. Applying the result to (4) we have $\xi=1, \boldsymbol{\omega}_{\mathrm{s}, \text { opt }}=0$ and any vector satisfying the amplitude constraint can serve as the optimum solution of $\boldsymbol{\omega}_{\mathrm{C}, \text { opt }}$.

Let's consider the GNSS signal acquisition using a uniform linear antenna array with 9 elements. Suppose there are multi-satellite signals and 2 narrow interferences in sight with the SNR and interference to noise ratio (INR) is $-20 \mathrm{~dB}$ and $50 \mathrm{~dB}$ respectively, while the signal incident direction, carrier frequency, PN code frequency and sampling frequency are 15 degrees, $48.16 \mathrm{MHz}, 10.23$ $\mathrm{MHz}$ and $65 \mathrm{MHz}$ respectively. Suppose the length of the local PN code vector is 4 and the 4th code is in phase with the satellite signal. Simulation results are shown in Fig.2. 

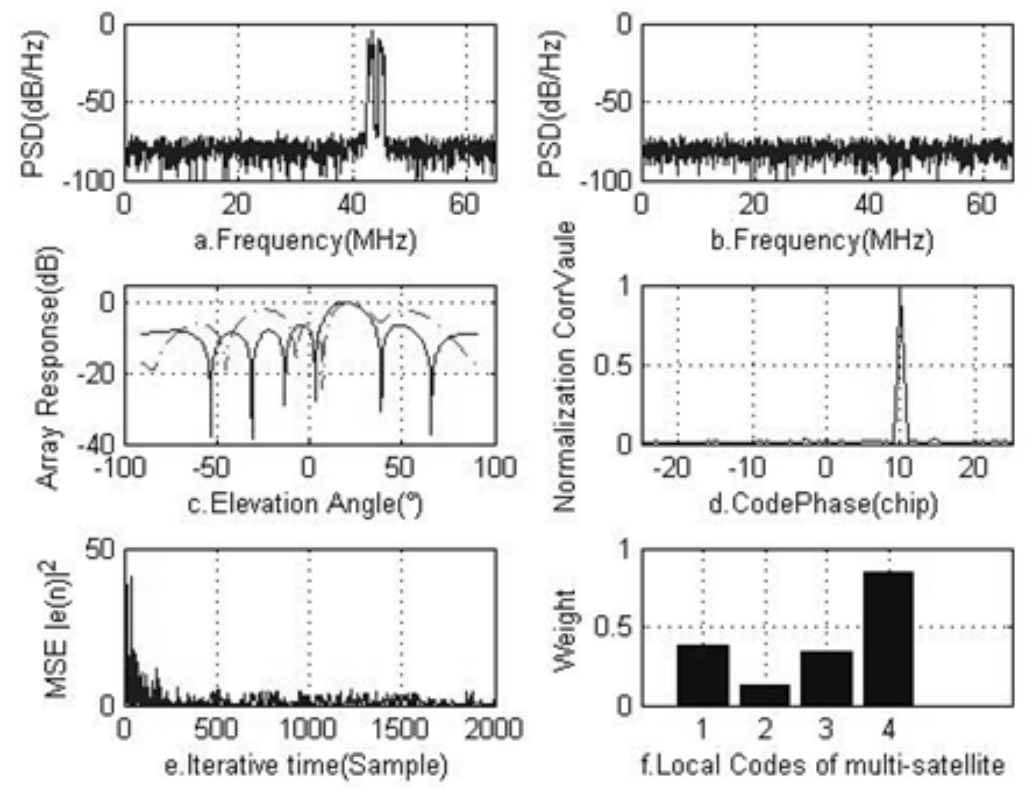

Fig. 2 Multi-satellite joint acquisition with interference mitigation simulation

a Signal power spectrum densities (PSD) received by antenna element

b Signal PSD after subspace projection

c Contrast between estimated DOA after beamforming and true DOA

d Acquisition correlation peak of satellite 4

e Convergent rate of the adaptive algorithm

f Local PN code weight after convergence

\section{Summary}

Through the above mathematic derivation in this letter, we find that when the local code delay and carrier Doppler are in phase with a satellite in view, after the adaptive filters are convergent will have a peak whose location corresponds to the PN code of the acquired satellite, and the main lobe of the beamformer will steer to its DOA. Using the proposed scheme all the GNSS satellites in view can be searched in a test bins and the acquisition can be clearly achieved in the low SNR and the strong interferences environment.

\section{References}

[1] H.L. Yang, W.R. Wu. 'A low-complexity adaptive antenna array code acquisition', Signal Processing, 2008, 88, pp. 1191-1202.

[2] H.L. Yang, W.R. Wu. 'A novel adaptive antenna array for DS/CDMA code acquisition', IEEE Trans. Signal Process. 2007, 55, (9), pp. 4567-4580.

[3] H.P., H.S., J.I. and P.L.. 'Serial search code acquisition using smart antennas with single correlator or matched filter’, IEEE Trans. Communication. 2008, 56, (2), pp. 299-308.

[4] B.Wang and H.Kwon. 'PN code acquisition for DS-CDMA System employing smart antenna', IEEE Trans. Wireless Communications, 2003, 2, (1), pp. 108-117.

[5] J.A., C.F., P.C., 'Antenna array based GNSS signal acquisition for interference mitigation', IEEE Trans. Aerospace and Electronic Systems. 2013, 49, (1), pp. 223-243.

[6] S.K., R.I.. 'STAP for GPS Receiver Synchronization', IEEE Trans. Aerospace and Electronic Systems. 2004, 40, (1), pp. 132-144. 